\title{
Article \\ Fibronectin 1B Gene Plays an Important Role in Loach Barbel Air-Breathing
}

\author{
Bing Sun ${ }^{1,+}$, Songqian Huang ${ }^{1,2,+}$, Longfei Huang ${ }^{3}$, Lijuan Yang ${ }^{1,3}$, Jian Gao ${ }^{1,3, *}$ and Xiaojuan Cao $1,3, *$ (1) \\ 1 Engineering Research Center of Green development for Conventional Aquatic Biological Industry in the \\ Yangtze River Economic Belt, Ministry of Education, College of Fisheries, Huazhong Agricultural University, \\ Wuhan 430070, China; sunbing931014@163.com (B.S.); huangsongqian0115@gmail.com (S.H.); \\ atyanglijuan@163.com (L.Y.) \\ 2 Department of Aquatic Bioscience, Graduate School of Agricultural and Life Sciences, \\ The University of Tokyo, Bunkyo, Tokyo 113-8657, Japan \\ 3 Key Lab of Freshwater Animal Breeding, Ministry of Agriculture, College of Fisheries, \\ Huazhong Agricultural University, Wuhan 430070, China; 15207173315@163.com \\ * Correspondence: gaojian@mail.hzau.edu.cn (J.G.); caoxiaojuan@mail.hzau.edu.cn (X.C.); \\ Tel.: +86-(027)-87282113 (J.G. \& X.C.) \\ + Means co-first author
}

check for updates

Citation: Sun, B.; Huang, S.; Huang, L.; Yang, L.; Gao, J.; Cao, X. Fibronectin 1B Gene Plays an Important Role in Loach Barbel Air-Breathing. Int. J. Mol. Sci. 2021, 22, 11928. https://doi.org/10.3390/ ijms222111928

Academic Editor: Carlo C. Lazado

Received: 6 September 2021

Accepted: 1 November 2021

Published: 3 November 2021

Publisher's Note: MDPI stays neutral with regard to jurisdictional claims in published maps and institutional affiliations.

Copyright: (c) 2021 by the authors. Licensee MDPI, Basel, Switzerland. This article is an open access article distributed under the terms and conditions of the Creative Commons Attribution (CC BY) license (https:// creativecommons.org/licenses/by/ $4.0 /)$.

\begin{abstract}
Loach (Misgurnus anguillicaudatus) is well known to perform air-breathing through the posterior intestine and skin. However, we find here for the first time a unique central vascular structure in the loach barbel, with a blood-gas diffusion distance as short as that of the posterior intestine. Under acute hypoxia, the distance of loach barbels became significantly shorter. Moreover, barbel removal significantly decreased air-breathing frequency of the loach. These findings imply that the barbel is another air-breathing organ of the loach. For further investigation of loach barbel air-breathing, a transcriptome analysis of barbels with air exposure treatment was performed. A total of 2546 differentially expressed genes (DEGs) between the T-XU (air exposure) and C-XU (control) group were identified, and 13 key DEGs related to barbel air-breathing were screened out. On this foundation, sequence, expression, and location analysis results indicated an important positive role of fibronectin $1 \mathrm{~b}(f n 1 b)$ in loach barbel air-breathing. We further generated an $f n 1 b$-depletion loach (MT for short) using the CRISPR/Cas 9 technique. It was indicated that depletion of $f n 1 b$ could weaker barbel air-breathing ability. In conclusion, due to nonlethal and regenerative characteristics, the loach barbel, a newly discovered and $f n 1 b$-related fish air-breathing organ, can be a good model for fish air-breathing research.
\end{abstract}

Keywords: Misgurnus anguillicaudatus; barbel; air-breathing; transcriptome; fn1b

\section{Introduction}

Since fish air-breathing is an important evolutionary adaptation for aquatic hypoxia, it has fascinated researchers for at least a century [1-3]. Up to now, about 400 species of fish have been reported to have the ability of assisted air-breathing [4]. There are several different fish air-breathing organs reported, including modified swim bladder, oropharyngeal cavity, skin, stomach, and intestine [3,4], which share similar features, such as being well-vascularized [5] and a short blood-gas diffusion distance [6], similar to the features of higher vertebrate lungs. It has been reported that the blood-gas diffusion distance of the intestine in Hoplosternum thoracatum is 1-2 $\mu \mathrm{m}$ [7], of the stomach in Ancistrus multispinis is $0.6 \mu \mathrm{m}$ [8], and of the intestine in Paramisgurnus dabryanus is $1.95 \mu \mathrm{m}$ [9]. Table S1 summarizes the distance of air-breathing organs in six air-breathing fish [7-12]. In general, the blood-gas diffusion distance of the assistant air-breathing organs in fish is less than $3 \mu \mathrm{m}[13,14]$.

So far, studies on air-breathing fish have mainly focused on histology, respiratory physiology, aquaculture, and evolutionary biology [15-18]. In recent years, along with the 
rapid development of sequencing, a very small number of studies have been performed on the transcriptome, microRNAome, and genome to reveal the molecular mechanisms of fish air-breathing formation and regulation [19]. However, all these studies remain at the stage of massive data filtering. In our previous study, a transcriptome analysis of the posterior intestine (an air-breathing organ) in loach (Misgurnus anguillicaudatus) with air-breathing inhibition was performed, focusing on the pathways of angiogenesis and vascular development [20]. Through comparative transcriptome analysis, some genes (for example, fibronectin $1(f n 1)$, integrin alpha (itga), and vascular endothelial growth factor $(v e g f))$ potentially related to air-breathing were screened out from four air-breathing fishes, namely, snakehead fish (Channa argus) [21], channel catfish (Ictalurus punctatus), blue catfish (I. furcatus), and their F1 hybrid [22]. Due to the slow development of in vivo gene editing research in air-breathing fish and the lack of good models of air-breathing organs (with nonlethal characteristics) in fish, there are almost no key genes related to air-breathing reported. Certainly, fish air-breathing molecular mechanisms cannot really be unveiled.

The loach M. anguillicaudatus, an important economic fish species in many Asian countries including China, Japan, and South Korea [23], has attracted the attention of scholars because of its air-breathing for many years [19,24]. With the aid of assisted airbreathing, the demersal loach can survive well in hypoxic environments. The loach must conduct air-breathing even in air-saturated water [25]. Taking it as a model, our laboratory has carried out studies on fish air-breathing mechanisms for more than 10 years $[19,20,26]$. The existing studies indicate that the loach has more than one assisted air-breathing organ (i.e., posterior intestine and skin), which is rare. Does the loach have other air-breathing organs to adapt to low oxygen environment? In other words, is there another organ with a rich capillary network and a short gas diffusion distance, similar to the posterior intestine and skin in the loach, which can conduct air-breathing? There is a viewpoint that the fish barbel is a subsidiary extended structure of the skin $[27,28]$. Therefore, can the developed barbels in the loach perform air-breathing?

In this study, we firstly compared the histological structures of barbels among loach M. anguillicaudatus (a water-air bimodal respiration fish), large-scale loach (P. dabryanus, a water-air bimodal respiration fish which can conduct air-breathing through its posterior intestine), zebrafish (Danio rerio, an aquatic breather), and yellow catfish (Pelteobagrus fulvidraco, an aquatic breather). Meanwhile, the barbel blood-gas diffusion distance and air-breathing frequency estimations, barbel excision, and acute hypoxia experiments were performed to examine the loach barbel air-breathing ability. Then, an air exposure experiment was carried out for barbel transcriptome analysis. Accordingly, sequence, expression, and location analyses were conducted to finally screen out the key air-breathing related gene, namely, fn $1 b$. At last, an $f n 1 b$-mutant loach was generated by CRISPR/Cas 9 for the first time to further confirm the role of $f n 1 b$ in loach barbel air-breathing. This study suggests that the loach barbel is a newly discovered fish air-breathing organ, and fn1b is closely involved in its air-breathing function. Due to the nonlethal and regenerative characteristics of fish barbels $[29,30]$ and the high efficiency of in vivo gene editing in loach $M$. anguillicaudatus [31], the loach barbel will be a good model for mechanistic investigations of fish air-breathing.

\section{Results}

\subsection{Histological Structures of Loach Barbels Suitable for Air-Breathing}

Two pairs of maxillary barbels (recoded as MB1 and MB2), one pair of rostal barbels (RB), and two pairs of submaxillary barbels (SB1 and SB2) were observed in loach $M$. anguillicaudatus (Figure 1A). In the loach, SB1 and SB2 were very short, while the other three barbels (i.e., MB1, MB2, and RB) were large. A linear regression analysis result demonstrated that there was a highly positive correlation between the average length of barbels (including MB1, MB2, and RB) and body length of the loach (Figure 1B). Figures 1C and S1 show that the five pairs of barbels in the loach (MB1, MB2, RB, SB1, and SB2) had similar histological structures. In addition to goblet cells, melanocytes, and taste buds, a large 
number of erythrocytes enriched in the central blood vessel and many capillaries, the structural basis of air-breathing, were found in the loach barbels. This was consistent with the transmission electron microscopy observation results (Figure 1D).
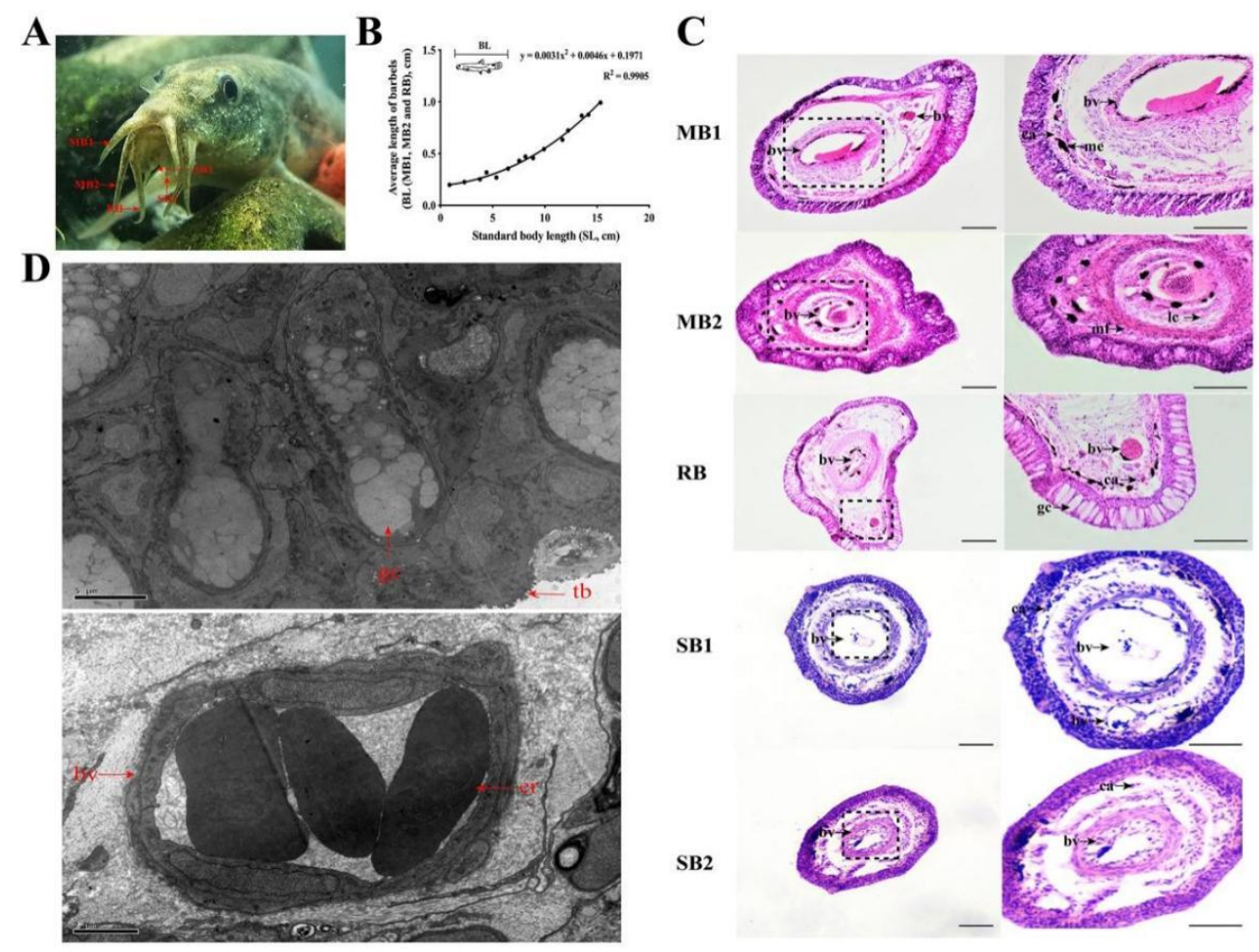

Figure 1. Morphological and histological observations of loach barbels. (A) Visual picture of loach barbels. (B) Linear regression curve of the average length of barbels including MB1, MB2, and RB (ordinate) and standard body length (SL, abscissa) in loach. (C) Observations of H\&E-stained transverse sections of loach barbels. The visual fields in the black dotted boxes are magnified to the right. The scale bars in the left and right columns of transverse sections of loach barbels are $30 \mu \mathrm{m}$ and $100 \mu \mathrm{m}$, respectively. (D) Transmission electron microscopy observation results of loach barbels. The scale bars of the top and bottom pictures are $5 \mu \mathrm{m}$ and $2 \mu \mathrm{m}$, respectively. $\mathrm{MB}$, maxillary barbel; RB, rostal barbel; SB, submaxillary barbel; H\&E, hematoxylin and eosin; bv, blood vessels; er, erythrocytes; ca, capillaries; $\mathrm{mf}$, muscle fiber; me, melanocytes; lc, loose connective tissue; gc, goblet cells; tb, taste buds.

There was an obvious difference in barbel histological structures between the loach and other three fishes (one water-air bimodal respiration fish and two aquatic breathers). Unlike the large blood vessel found in the loach barbel center, cartilage tissues existed in the centers of barbels from the other three fishes including the large-scale loach, zebrafish, and yellow catfish (Figure 2A). Figure 2B shows no significant difference in blood-gas diffusion distance between the barbel $(2.495 \pm 0.194 \mu \mathrm{m})$ and posterior intestine $(2.488 \pm 0.0997 \mu \mathrm{m})$ of the loach, suggesting that loach barbels are suitable for air-breathing to some extent. After removing the barbels, the movement of the loach became slow. The air-breathing frequency of the loach with barbel excision was significantly lower than that of the wildtype (WT) loach (Figure 2C). However, there was no significant difference in air-breathing frequency between the WT and barbel-excised large-scale loach (Figure 2D), and barbel excision had no effect on its movement. 
$\mathbf{A}$

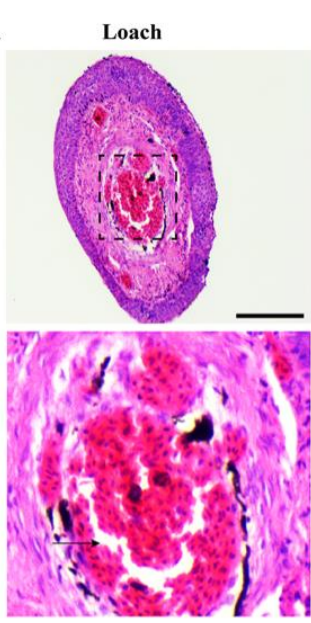

B

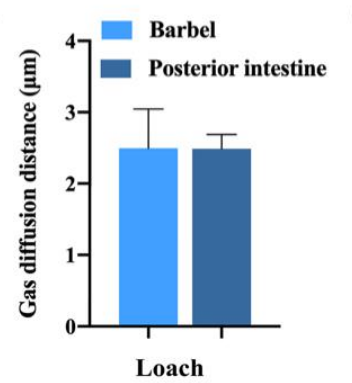

Large-scale loach
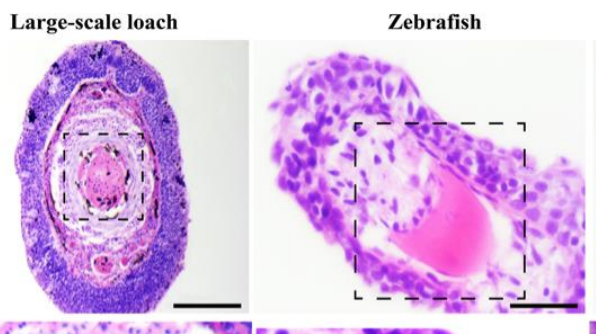

Zebrafish
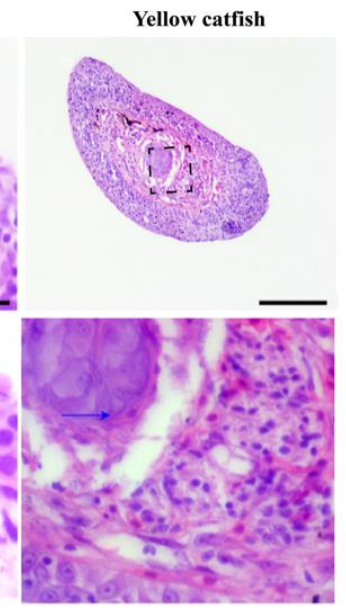

C

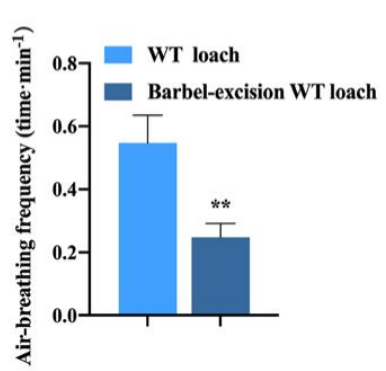

D

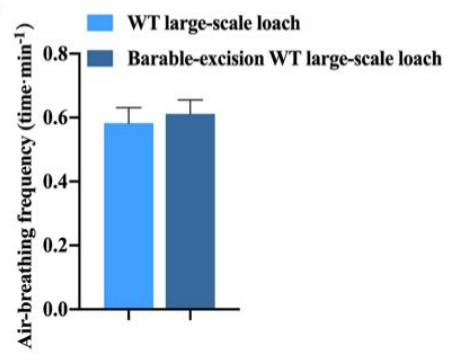

Figure 2. Comparisons of barbel histological structures, gas diffusion distances, and air-breathing frequency. (A) Comparison of histological structures of barbels among loach, large-scale loach, zebrafish, and yellow catfish. The visual fields in black dotted boxes are magnified below. The black arrow indicates erythrocytes, and the blue arrows indicate cartilage tissues. Scale bar $=100 \mu \mathrm{m}$. (B) Comparison of gas diffusion distance between the barbel and posterior intestine of loach. (C) Comparison of air-breathing frequency between WT and barbel-excised loach. (D) Comparison of air-breathing frequency between WT and barbel-excised large-scale loach. WT, wild-type. ** very significant difference $(p<0.01)$.

\subsection{The Changes in Histological Structures and Gas Diffusion Distances of Loach Barbels in Response to Acute Hypoxia}

Along with the increase in acute hypoxia treatment time, the capillaries of loach barbels and posterior intestines extended to the dermal layer and mucosal layer, respectively (Figure 3A). For loach barbels, the blood-gas diffusion distance at 10 and $20 \mathrm{~min}$ post hypoxia (mph) was significantly lower than that without hypoxia treatment (i.e., at $0 \mathrm{mph}$ ) (Figure 3B). Similarly, the gas diffusion distance in the posterior intestine at $20 \mathrm{mph}$ was significantly lower than that at 0 and $10 \mathrm{mph}$ (Figure 3C).

\subsection{Transcriptome Analysis of Loach Barbels with Air Exposure}

After assembly of the mapped reads of six cDNA libraries, a total of 250.54 million raw reads and 247.26 million clean reads were obtained. The percentage of mapped clean reads ranged from $82.82 \%$ to $85.82 \%$. The Q30 values of more than $93.32 \%$ indicated a high quality of the sequencing data (Table S2). 
A WT Loach

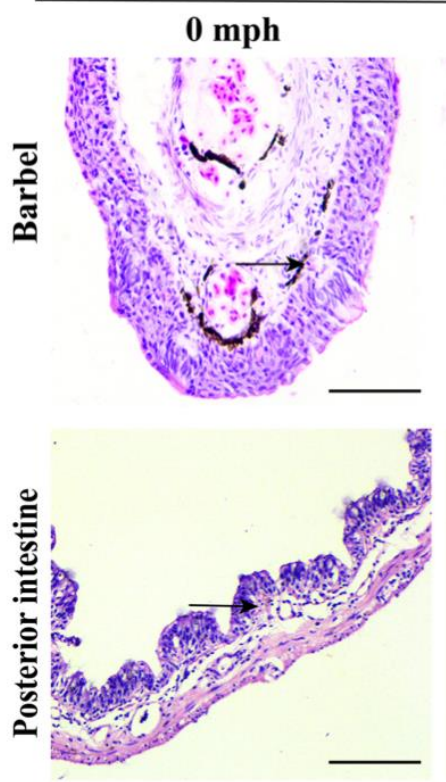
$10 \mathrm{mph}$
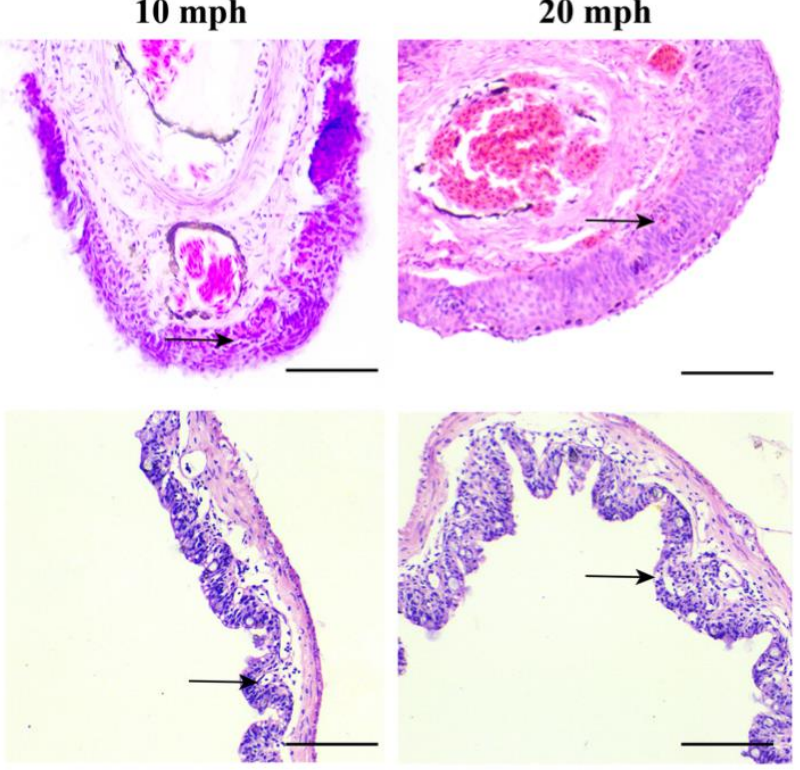

B

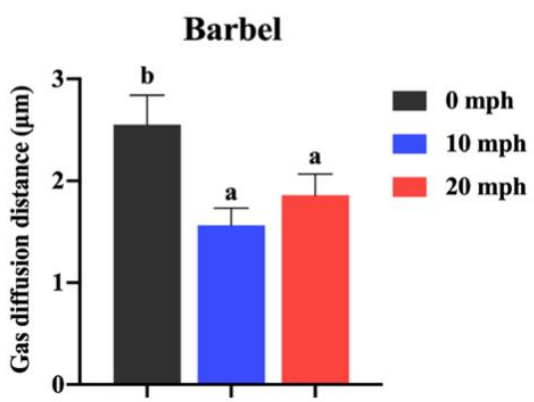

C

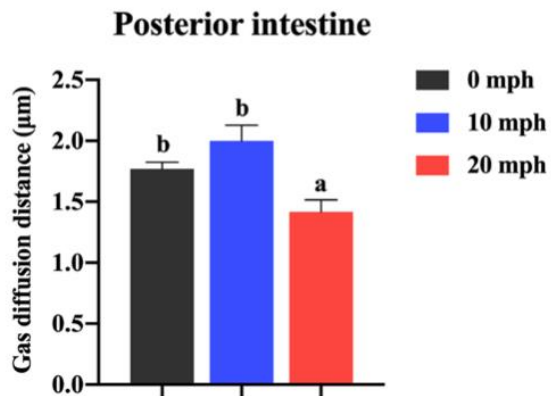

Figure 3. Histological structures and gas diffusion distance of barbels and posterior intestines of loach with acute hypoxia treatment. (A) Histological structures of barbels and posterior intestines of loach with acute hypoxia treatment. $\mathrm{mph}$, minute post hypoxia. Black arrows indicate capillaries. Scale bar $=100 \mu \mathrm{m}$. (B,C) The gas diffusion distances of barbels (B) and posterior intestines (C) of loach with acute hypoxia treatment. Different letters above error bars indicated significant a difference among different groups $(p<0.05)$.

2.3.1. Gene Ontology (GO) and Kyoto Encyclopedia of Genes and Genomes (KEGG) Enrichment Analysis of Differentially Expressed Genes (DEGs)

A total of 2546 DEGs were identified, with 1145 upregulated and 1401 downregulated in the air exposure group (T-XU group) in comparison to the control group (C-XU group, without air exposure) (Figure 4A, Figure S2A). The GO analysis showed that all DEGs were classified into three categories: biological process, cellular component, and molecular function, involving 24, 15, and $11 \mathrm{GO}$ terms, respectively (Figure S2B). The top 20 significantly enriched GO terms are summarized in Figure 4B. Furthermore, KEGG analysis showed that the 2546 DEGs belonged to 33 pathways, which included signal transduction (324 DEGs; $12.73 \%$ ), immune system (183 DEGs; 7.19\%), endocrine system (166 DEGs; 6.32\%), and transport and catabolism (132 DEGs; 5.18\%) (Figure S2C). Among these pathways, the top 20 significantly enriched KEGG pathways were identified (Figure 4C). 
A

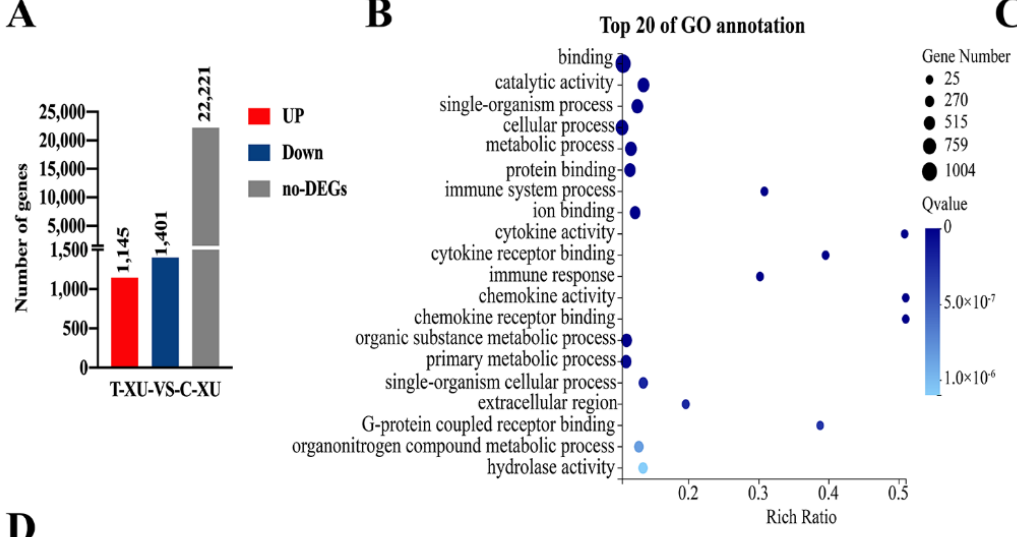

D

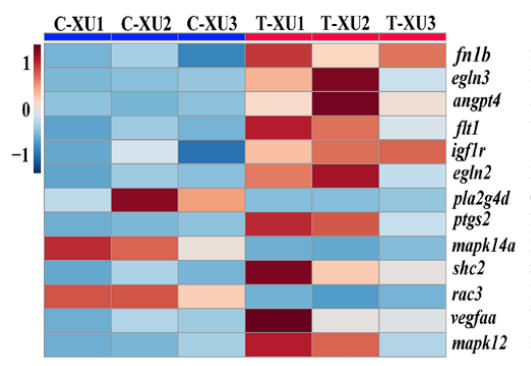

\section{Description}

fibronectin 1

Egl nine homolog Insulin-like growth factor 1 receptor Egl nine homolog 2 Cytosolic phospholipase A2 delta Prostaglandin G/H synthase 2
Mitogen-activated protein kinase $14 \mathrm{~A}$ Mitogen-activated protein kinase
SHC-transforming protein 2 SHC-transformin
Ras-related C3

Vascular endothelial growth factor A-A Mitogen-activated protein kinase 12
C

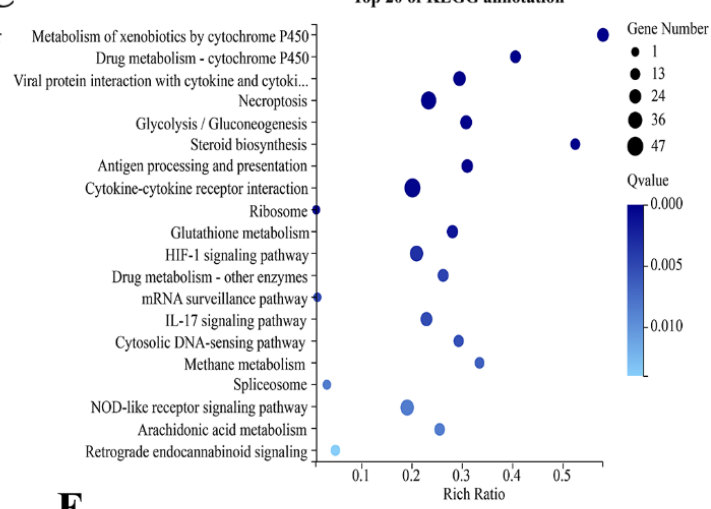

$\mathbf{E}$
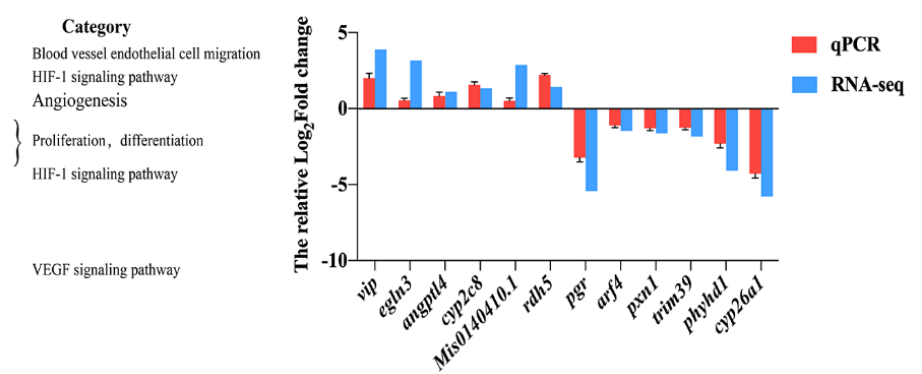

Figure 4. Identification and functional annotations of DEGs in barbels between T-XU and C-XU group (T-XU vs. C-XU). (A) Numbers of upregulated, downregulated, and non-DEGs. (B) Top 20 GO terms in DEGs. (C) Top 20 KEGG pathways in DEGs. (D) Heat map of expression levels of 13 key DEGs related to air-breathing. (E) qPCR validation of RNA-seq data. DEGs, differentially expressed genes. T-XU and C-XU represent the air exposure group and control group, respectively. qPCR, quantitative PCR. vip, VIP intestinal peptide; egln3, egl nine homolog 3; angptl4, angiopoietin-related protein 4; cyp2c8, cytochrome P450 2C8; rdh5, 11-cis-retinol dehydrogenase; $p g r$, progesterone receptor; arf4, ADP-ribosylation factor 4; pxn1, pentraxin fusion protein precursor; trim39, E3 ubiquitin-protein ligase TRIM39; phyhd1, phytanoyl-CoA dioxygenase domain-containing protein 1; cyp26a1, cytochrome P450 26A1.

\subsubsection{Mining of DEGs Related to Barbel Air-Breathing}

Referring to the structural needs of air-breathing function in fish (namely, rich capillary networks) and the existing research results [19,21,22], some KEGG pathways of DEGs including the HIF-1 signaling pathway, VEGF signaling pathway, and blood vessel endothelial cell migration pathway were targeted, and 13 key DEGs $(f n 1 b$, egl nine homolog 3 (egln3), angiopoietin 4 (angpt4), vascular endothelial growth factor receptor $1(f l t 1)$, insulin-like growth factor 1 receptor (igflr), egl nine homolog 2 (egln2), cytosolic phospholipase A2 delta (pla2g4d), prostaglandin G/H synthase 2 (ptgs2), mitogen-activated protein kinase 14 a (mapk14a), shc-transforming protein 2 (shc2), ras-related C3 (rac3), vegfaa, and mitogen-activated protein kinase 12 (mapk12); 10 up- and three downregulated genes) were screened out (Figure 4D).

\subsection{3. qPCR Validation of RNA-Seq Data}

To validate the RNA-seq data, we randomly selected 12 DEGs to conduct quantitative PCR (qPCR) analysis. The qPCR results were consistent with the RNA-seq data (Figure 4E).

\subsection{Sequence and Expression Profiles of Fn1b in Loach}

To further confirm the role of $f n 1 b$ (the most prominent key DEG screened out above) in loach barbel air-breathing, a sequence analysis of FN1 was firstly performed. The FN1 conserved domains of loach, zebrafish, snakehead fish, and rice-field eel were predicted, showing that the FN1a protein was conserved in fish, while the FN1b protein of loach (with one FN3 module less) was very different from the other three fishes (Figure S3). This suggested that the $f n 1 b$ gene is special in loach. 
Next, Figure 5A,B show that the expression level of $f n 1 b$ in the air-breathing organ (oropharyngeal cavity in rice-field eel and suprabranchial organ in snakehead fish) was significantly higher than that of the aquatic breathing organ (gill). In addition, $f n 1 b$ was also found highly expressed in the posterior intestine (an air-breathing organ) of largescale loach (Figure 5C). These results suggest that $f n 1 b$ may be highly expressed in many air-breathing organs in fish. Compared with large-scale loach (air-breathing fish), zebrafish (aquatic breathing fish), and yellow catfish (aquatic breathing fish), the loach presented an obviously higher expression of $f n 1 b$ in the barbels (Figure 5C). In addition, compared with the kidney, about 68-, 118-, and 167-fold higher fn1b expression was detected in the skin, fin, and barbel (air-breathing organs) of loach, respectively (Figure 5D). This indicated that fn1b plays a positive role in loach barbel air-breathing.

A

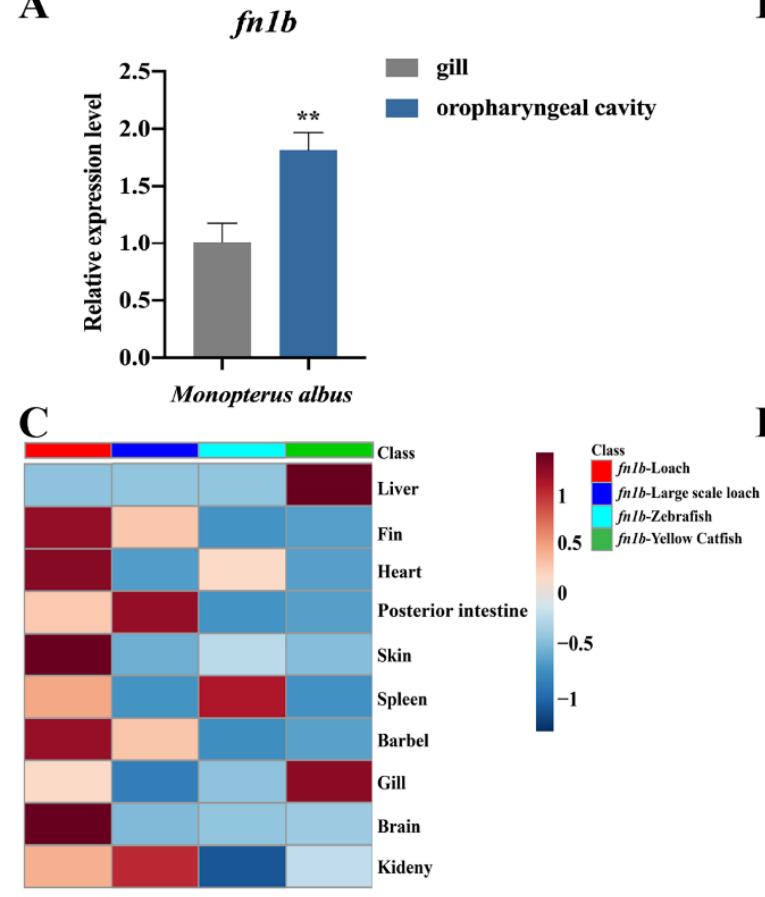

B
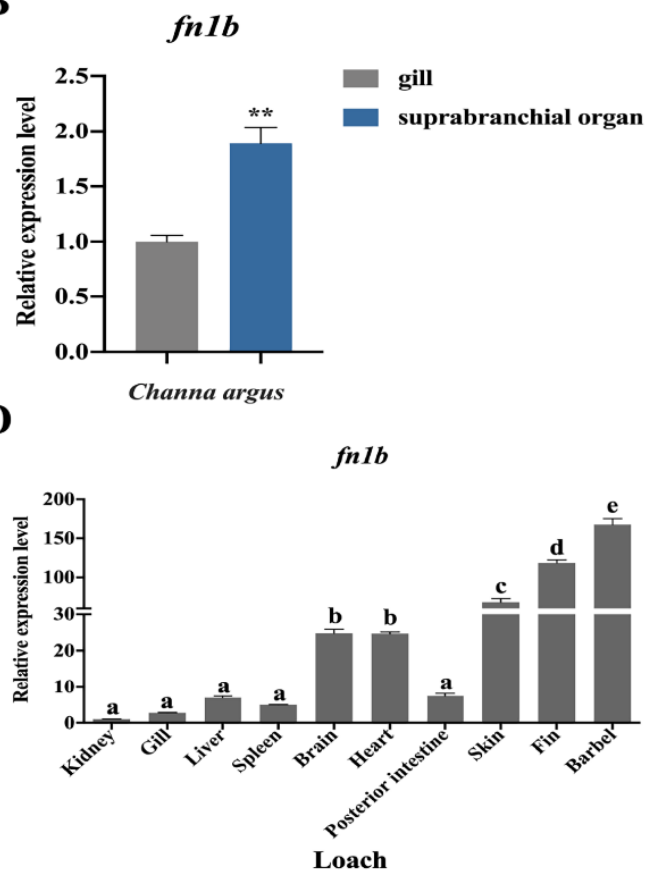

Figure 5. $f n 1 b$ expression levels. (A) Expression levels of $f n 1 b$ in the gill (aquatic breathing organ) and oropharyngeal cavity (an air-breathing organ) of rice-field eel Monopterus albus were calculated using the $2^{-\Delta \Delta C T}$ method. $\beta$-actin was used as the reference gene for normalization. (B) Expression levels of $f n 1 b$ in gill and suprabranchial organ (an air-breathing organ) from snakehead fish Channa argus were calculated using the $2^{-\Delta \Delta C T}$ method. $\beta$-actin was used as the reference gene for normalization. (C) Tissue expression levels of $f n 1 b$ in loach, large-scale loach, zebrafish, and yellow catfish. MetaboAnalyst5.0 was used for constructing the heat map. (D) Comparison of $f n 1 b$ relative expression across different tissues from the loach (Misgurnus anguillicaudatus). Tissue expression levels of $f n 1 b$ in loach were calculated using the $2^{-\Delta \Delta C T}$ method. $\beta$-actin and gapdh were used as reference genes for normalization. fn $1 b$, fibronectin $1 \mathrm{~b}$; gapdh, glyceraldehyde-3phosphate dehydrogenase. Different letters above error bars indicate significant difference among different tissues $(p<0.05)$. ** very significant difference $(p<0.01)$.

Then, whole-mount in situ hybridization (WISH) and tissue in situ hybridization (TISH) were performed to locate the $f n 1 b$ gene in loach. Figure $6 \mathrm{~A}$ showed that $f n 1 b$ was highly expressed in the polster, tail bud, and external yolk syncytial layer of loach embryos at $12 \mathrm{~h}$ post fertilization (hpf), in the eye and brain of loach embryos at $24 \mathrm{hpf}$, and in the lip and barbel of the loach at 5 days post fertilization (dpf). At $12 \mathrm{dpf}$, the expression signals were mainly concentrated in the barbel, jaw, and heart. Furthermore, the TISH results indicated that $f n 1 b$ was located in blood vessels of loach barbels (Figure 6B). 
A
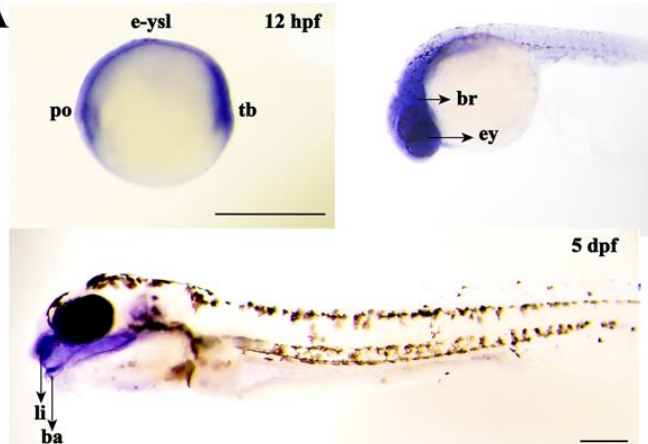

B

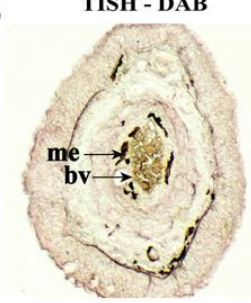

2 hpf

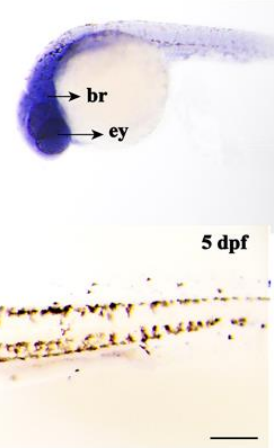

TISH - DAB

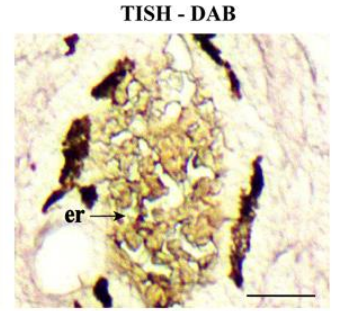

24 hpf
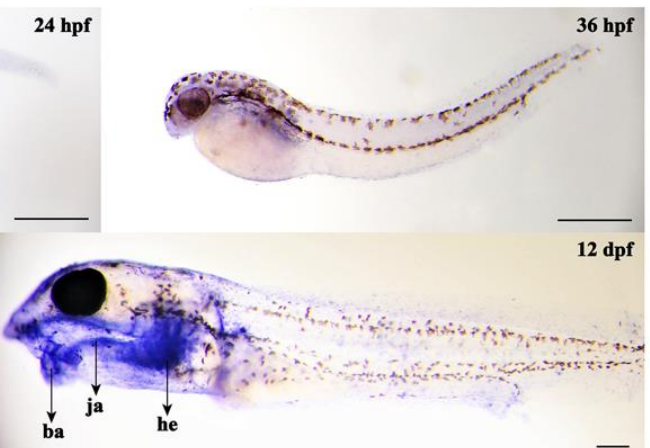

TISH - DAB + H\&E

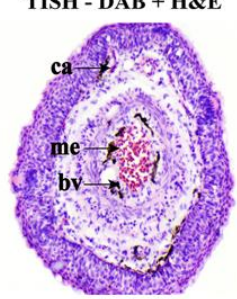

TISH - DAB + H\&E

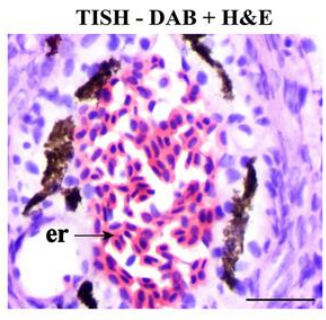

Figure 6. The localization of $f n 1 b$ in loach. (A) Whole-mount in situ hybridization of $f n 1 b$ in loach embryos of different developmental stages, including 12 hpf, 24 hpf, 36 hpf, 5 dpf, and 12 dpf. hpf, hours post fertilization; dpf, days post fertilization; po, polster; tb, tail bud; e-ysl, external yolk syncytial layer; br, brain; ey, eye; li, lip; ba, barbels; he, heart; ja, jaw. Scale bar = $1 \mathrm{~mm}$. (B) Tissue in situ hybridization (TISH) of $f n 1 b$ in loach barbels. Brown color signifies the signals. $\mathrm{DAB}, 3,3^{\prime}$-diaminobenzidine tetrahydrochloride; H\&E, hematoxylin and eosin; bv, blood vessel; me, melanocytes; er, erythrocytes; ca, capillary. The scale bars of the left and the right pictures indicated $100 \mu \mathrm{m}$ and $10 \mu \mathrm{m}$, respectively.

\subsection{The Depletion of Fn1b Weakened the Air-Breathing of Loach Barbel}

The chromatogram of MT loach $\left(f n 1 b^{+/-}\right.$loach) showed that a C base was missing at exon 3 of the $f n 1 b$ gene, which led to premature translation termination (the original translation of 2398 amino acids was terminated at the 135th) (Figure S4A). The survival rate of F2 generation fertilized eggs (F1 generation self-crossed) was obviously lower than that of F1 generation (F0 generation crossed with WT loach) and WT loach fertilized eggs (Figure S4B), which to some extent could explain the failure of $f n 1 b^{-/}$generation in this study. Figure S4C shows that the expression level of $f n 1 b$ in MT loach was significantly lower than that of WT loach.

Figure 7A shows the histological structures of barbels of WT and MT loach. It is worth noting that the barbel blood-gas diffusion distance of MT loach was significantly longer than that in WT (Figure 7B). Under chronic hypoxia, we found that the movement of MT loach was relatively slow compared with WT loach. Figure 8A indicates that the water oxygen consumption of MT loach with chronic hypoxia treatment was clearly lower than that of WT loach. Meanwhile, the air-breathing frequency and the asphyxia point of MT loach were significantly lower than those of WT loach (Figure 8B,C). 
$\mathbf{A}$

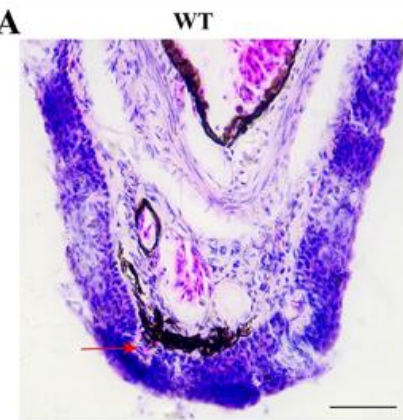

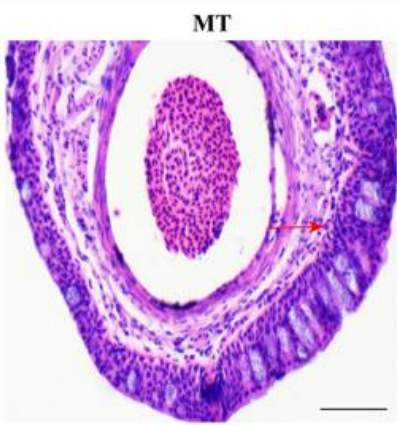

B

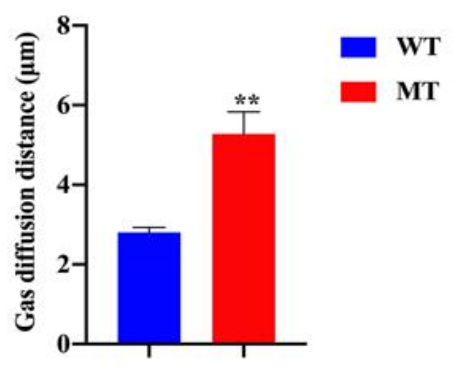

Figure 7. Histological structures (A) and gas diffusion distances (B) of barbels in WT and MT loach. Red arrows show capillaries. WT, wild-type loach; MT, fn1b-depletion loach. Scale bar $=100 \mu \mathrm{m}$. ** very significant difference $(p<0.01)$.

A

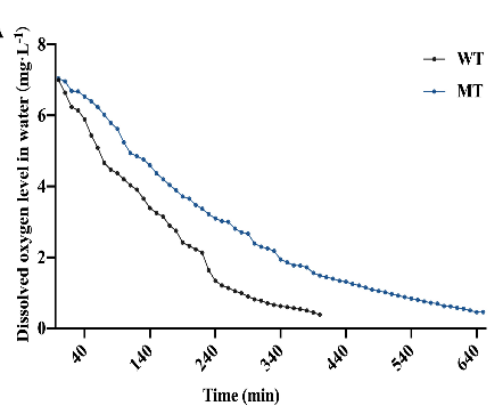

B

B

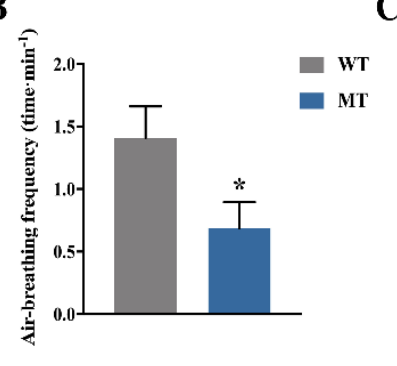

C

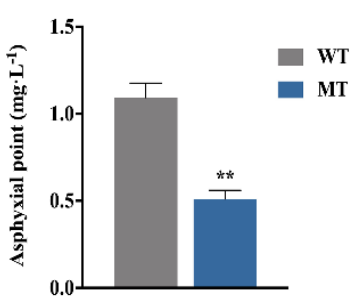

Figure 8. The effects of fn1b-depletion on air-breathing function of WT and MT loach under chronic hypoxia. (A) Water dissolved oxygen levels in the sealed conical flasks with WT and MT loach. $(\mathbf{B}, \mathbf{C})$ Comparison of air-breathing frequency (B) and asphyxia point (C) between WT and MT loach under chronic hypoxia. WT, wild-type loach; MT, fn1b-depletion loach. * significant difference $(p<0.05)$; ** very significant difference $(p<0.01)$.

\section{Discussion}

Many fish have barbels. The number of barbels in fish varies from species to species. For example, Osteogeniosus bleeker [32] has only one pair of barbels. Both common carp (Cyprinus carpio) [33] and zebrafish [28] possess two pairs of barbels. This study shows that the number of barbels in loach is up to five pairs. The fish barbel is generally considered as a sensory organ, which fish can use to find and choose food. The barbel structures are simple, normally composing of a central rod of cartilage, connective tissue, taste buds, and nerve trunk [30]. Interestingly, in this study, we found a unique central vascular structure in loach barbel, very different from the axial cartilage tissues in other aquaticbreathing and air-breathing fishes. Loach barbels are well-vascularized, which is the structural basis of air-breathing [34]. In addition, the blood-gas diffusion distance of the barbel was as short as that of the posterior intestine (a confirmed air-breathing organ) in loach. The blood-gas diffusion distance is an important indicator of air-breathing [3]. A short blood-gas diffusion distance is a structural adaptation of air-breathing organs, which can greatly enhance the efficiency of gas exchange [34]. Under acute hypoxia, the blood-gas diffusion distance of loach barbels became significantly shorter, and this was also the case for the loach posterior intestine, as well as the air-breathing organs of H. thoracatum [7] and Boleophthalmus boddaerti [35]. After removing barbels, a significant decrease was observed in loach air-breathing frequency. Considering these characteristics of loach barbels above (namely, the unique vascular structures, short blood-gas diffusion distance, and air-breathing behavior), it is reasonable to believe that the loach barbel is a newly discovered air-breathing organ.

Currently, transcriptome analysis is widely used to explore molecular mechanisms of important life activities [36,37] In this study, a transcriptome analysis of loach bar- 
bels with air exposure treatment was performed, and some KEGG pathways and key genes related to barbel air-breathing were screened out. Among them, the angiogenesis and vascular development pathway, HIF-1 signaling pathway, and VEGF pathway were prominent. These pathways were also identified in previous studies on air-breathing of loach [19] and snakehead fish [21]. In this study, the blood vessel morphogenesis- and development-related genes (for example, $f n 1 b$ and vegfaa) were remarkably induced in loach barbels with air exposure, suggesting that $f n 1 b$ and vegfaa may be closely related to fish air-breathing organs.

It has been reported that $f n 1$ is crucial for blood vessel development and heart morphogenesis in both zebrafish [38] and mouse [39,40]. In this study, fn1b showed a significantly high expression in loach barbels based on qPCR and ISH analysis. Moreover, the expression levels of $f n 1 b$ in air-breathing organs of rice-field eel and snakehead fish were significantly higher than in gills, as also found in a previous study reported by Jiang et al. [21]. Therefore, these results further indicated that $f n 1 b$ is important for loach barbel air-breathing.

For exploring the role of $f n 1 b$ in air-breathing of loach barbels, an $f n 1 b$-mutant loach line was generated for the first time using the CRISPR/Cas9 technique. Compared with WT loach, MT loach possessed a longer blood-gas diffusion distance in barbels, suggesting that $f n 1 b$ depletion weakened the air-breathing ability of loach barbels. In addition, under chronic hypoxia condition, the oxygen consumption, air-breathing frequency, and asphyxia point of MT loach were significantly decreased compared with WT loach. As we know, oxygen consumption is positively correlated with fish movement [41,42]. The low oxygen consumption and air-breathing frequency in MT loach would lead to its weakened airbreathing ability, so as to maintain slow movement. These findings again suggested that $f n 1 b$ plays a positive role in barbel air-breathing of loach.

Reports related to the fish $f n 1$ gene and its encoded fibronectin (FN) were mainly generated in zebrafish. There are two subtypes of $f n 1 a$ and $f n 1 b$ in zebrafish [43] and loach. Interestingly enough, unlike other fish species (including zebrafish, rice-field eel, and snakehead fish), the FN1b protein of loach lacks an FN3 module. FN3 is the most abundant and common domain of the FN1 protein, which has important functional and structural properties in angiogenesis [44]. The special structure of loach FN1b suggests that it may perform different functions, which needs further investigations.

In conclusion, the present study strongly suggests that barbels play an important role as air-breathing organs, which has never been identified, particularly in loach. Moreover, using loach barbel as a new model for air-breathing in fishes, we clearly show that fn1b plays a crucial role in this process. As we propose the barbel as an additional air-breathing organ in fish, there are even more important gene analysis studies to conduct (fibronectin supramolecular complex) in sensory cells (electrophysiological responses of barbel cells to air or airborne molecules). These new perspectives opened up by our work are particularly important when it comes to further characterization of the functional importance of barbels in sensing the complex environment of a fish, such as hypoxia.

\section{Materials and Methods}

\subsection{Fish Species}

All experimental diploid WT loaches M. anguillicaudatus (body length of 10-12 cm; body weight of 9-10 g; 1 year old) were obtained from the Aquaculture Base of College of Fisheries, Huazhong Agricultural University in Wuhan City, Hubei Province, China. Large-scale loach (11-12 cm; 13-15 g; 1 year old), yellow catfish (10-13 cm; 110-130 g; 1 year old), rice-field eel (Monopterus albus, a water-air bimodal respiration fish which can conduct air-breathing through its oropharyngeal cavity; $18-20 \mathrm{~cm}$; 150-170 g; 1 year old), and snakehead fish (a water-air bimodal respiration fish which can conduct air-breathing through its suprabranchial organ; $19-21 \mathrm{~cm} ; 240-280 \mathrm{~g} ; 1$ year old) were obtained from the Baishazhou aquatic products market. Zebrafish (AB strain, $0.4-0.5 \mathrm{~g}$; $4-6 \mathrm{~cm} ; 2$ months old) were purchased from the Institute of Hydrobiology, Chinese Academy of Sciences, China Zebrafish Resource Center, Wuhan, China. 


\subsection{Morphological and Histological Observations and Estimations of Blood-Gas Diffusion Distance and Air-Breathing Frequency}

Morphological observation of WT loach barbels was performed, and the correlation of barbel length and body length was estimated. Barbels sampled from WT loach, largescale loach, zebrafish, and yellow catfish were fixed in $4 \%$ paraformaldehyde for $24 \mathrm{~h}$, dehydrated in graded ethanol, and embedded in paraffin wax. Cross-sections of $5 \mu \mathrm{m}$ thickness were stained with hematoxylin and eosin (H\&E) and then prepared for light microscopy, according to the method of Cao and Wang [45]. Next, the blood-gas diffusion distance, the shortest distance between the capillary and the epidermis, was measured with the use of Image J software (National Institutes of Health, Bethesda, MD, USA). Meanwhile, $\mathrm{H} \& \mathrm{E}$ staining of the posterior intestine tissue section of the loach was performed for blood-gas diffusion distance assessment.

For transmission electron microscopy, the barbel tissues (MB1, size: $1.0 \mathrm{~mm} \times 1.0 \mathrm{~mm}$ $\times 1 \mathrm{~mm}$ ) were fixed immediately in fixative, post-fixed in osmium tetroxide, and embedded in Spurr's resin. The section was performed by the platform of transmission electron microscopy of Huazhong Agricultural University. The microphotograph was taken using a transmission electron microscope (HT7700, HITACHI, Tokyo, Japan).

Three WT loaches and three barbel-excised loaches of the same size were placed into a $2 \mathrm{~L}$ conical flask containing $1.5 \mathrm{~L}$ of water (initial dissolved oxygen (DO): $6.0 \mathrm{mg} / \mathrm{L}$ ) (Figure S5A, normoxia treatment) to measure the air-breathing frequencies, which were recorded within $2 \mathrm{~h}$. At the same time, the swimming behaviors of the loaches were observed. Three parallel experiments were carried out. Similarly, the air-breathing frequencies of healthy WT and barbel-excised large-scale loach were measured.

To further explore the loach barbel air-breathing ability, an acute hypoxia experiment (Figure S5B) was performed. WT loaches were placed into $2 \mathrm{~L}$ conical flasks containing $1 \mathrm{~L}$ of water, whilst nitrogen was poured into the flasks to drastically decrease its dissolved oxygen content. Loach barbels and posterior intestines were sampled at 0,10 , and $20 \mathrm{mph}$ for histological analysis. Then, the blood-gas diffusion distances of both kinds of tissues were calculated.

\subsection{Transcriptome Analysis of Loach Barbel}

\subsubsection{Air Exposure Experiment}

A total of 18 WT loaches were used here. The loaches were cultured in a $25 \mathrm{~L}$ glass tank with $20 \mathrm{~L}$ of water (temperature: 24.0 to $26.0^{\circ} \mathrm{C}$; $\mathrm{pH}: 7.0$ to 7.5 ; DO: $6.0 \mathrm{mg} / \mathrm{L}$ ). Among them, nine loaches were randomly selected for barbel sampling, namely, the control group (C-XU). Then, the remaining nine loaches were placed on moist towels (air exposure group (T-XU), Figure S5C). Based on our pre-test results, an air exposure time of $6 \mathrm{~h}$ was applied. Barbels sampled from C-XU and T-XU group were stored at $-80{ }^{\circ} \mathrm{C}$ for RNA isolation.

\subsubsection{RNA Isolation and cDNA Library Constructions}

Total RNA was isolated from the barbel tissues using TRIzol Reagent (TaKaRa, Dalian, China) according to the manufacturer's protocol. The extracted RNA samples of high quality were used for cDNA synthesis. Poly (A) mRNA was isolated using oligo-dT beads (Qiagen). All mRNA was sheared into short fragments (200 nt) by adding fragmentation buffer. First-strand cDNA was generated using random hexamer-primed reverse transcription, followed by the synthesis of the second-strand cDNA using RNase H and DNA polymerase I. The cDNA fragments were purified using a QIAquick PCR extraction kit. These purified fragments were then washed with EB buffer for end reparation poly (A) addition and ligated to sequencing adapters. Following agarose gel electrophoresis and extraction of cDNA from gels, the cDNA fragments $(200 \pm 5 \mathrm{bp})$ were purified and enriched by PCR to construct the cDNA library (PE100). Six cDNA libraries were constructed (namely, C-XU-1, C-XU-2, C-XU-3, T-XU-1, T-XU-2, and T-XU-3) in this study. 


\subsubsection{Sequencing and Read Mapping}

The cDNA libraries were sequenced on an Illumina platform (Illumina Hiseq xten/NovaSeq6,000 sequencer Illumina, San Diego, CA, United States) and 150 bp pairedend reads were generated. The raw paired-end reads were trimmed and quality-controlled using SeqPrep (https:/ / github.com/jstjohn/SeqPrep, accessed on 11 August 2021) and Sickle (https:/ / github.com/najoshi/ sickle, accessed on 11 August 2021) with default parameters. Then, clean reads were separately aligned to the loach genome (unpublished) using HISAT2 (http:/ / www.ccb.jhu.edu/software/hisat, accessed on 11 August 2021) [46] software. The mapped reads of each sample were assembled by StringTie (https:/ / ccb. jhu.edu/software/stringtie/index.shtml? $\mathrm{t}=$ example, accessed on 11 August 2021) in a reference-based approach [47].

\subsubsection{Identification of DEGs and Functional Annotations}

To identify DEGs between the two different groups (T-XU and C-XU), the expression level of each transcript was calculated according to the transcripts per million reads (TPM) method. RSEM (http:/ / deweylab.biostat.wisc.edu/rsem/, accessed on 11 August 2021) [48] was used to quantify gene abundances. Differential expression analysis was performed using DESeq2. Genes with $\mid \log _{2}$ fold change $\mid \geq 1$ and false discovery rate (FDR) $<0.05$ were considered to be significantly differentially expressed genes [49]. In addition, GO functional enrichment analysis and KEGG pathway analysis were carried out using Goatools (https:/ / github.com/tanghaibao/Goatools, accessed on 11 August 2021) and KOBAS (http:/ / kobas.cbi.pku.edu.cn/home.do, accessed on 11 August 2021) [50].

\subsection{5. qPCR Validation of Transcriptome Data}

To examine the reliability of the transcriptome results, we randomly selected 12 DEGs (six upregulated genes: VIP intestinal peptide (vip), egln3, angiopoietin-related protein 4 (angptl4), cytochrome P450 2C8 (cyp2c8), mis0140410.1, and 11-cis-retinol dehydrogenase $(r d h 5)$; six downregulated genes: progesterone receptor $(p g r)$, ADP-ribosylation factor 4 (arf4), pentraxin fusion protein precursor ( $p \times n 1)$, E3 ubiquitin-protein ligase TRIM39 (trim39), phytanoyl-CoA dioxygenase domain-containing protein 1 (phyhd1), and cytochrome P450 26A1 (cyp26a1)) to conduct qPCR analysis. Total RNAs of the barbel samples from C-XU and T-XU group were extracted using RNA isoPlus (TaKaRa, Dalian, China). qPCR was carried out on a QuantStudioTM 3 Real-time PCR system (ThermoFisher) according to the manufacturer's instructions. qPCR conditions were as follows: $95^{\circ} \mathrm{C}$ for $30 \mathrm{~s}$ followed by 40 cycles consisting of $95^{\circ} \mathrm{C}$ for $5 \mathrm{~s}$ and $57^{\circ} \mathrm{C}$ for $30 \mathrm{~s}$. The fluorescent flux was then recorded, and the reaction continued at $72{ }^{\circ} \mathrm{C}$ for $6 \mathrm{~s}$ and $95{ }^{\circ} \mathrm{C}$ for $5 \mathrm{~s}$. Finally, the relative expression levels were normalized to the endogenous control gene $\beta$-actin and glyceraldehyde-3-phosphate dehydrogenase (gapdh) and calculated using the $2^{-\Delta \Delta C T}$ method. All procedures were based on the methods described by Liu et al. [51]. The relative expression levels of these genes ( $\log _{2}$ fold change (T-XU vs. C-XU)) observed by qPCR and RNA-seq analyses were used for graphical presentation. All of the primer sequences for qPCR are listed in Table S3.

\subsection{Sequence, Expression, and Location Analyses of fn1b}

\subsubsection{Sequence Analysis}

To further confirm the role of $f n 1 b$ gene (a key DEG screened out from the transcriptome analysis above) in loach barbel air-breathing, a sequence analysis was firstly conducted. Deduced amino-acid sequences of $f n 1$ genes in three other fish species (zebrafish, rice-field eel, and snakehead fish) were obtained from the NCBI database. The conserved domains were analyzed using Conserved Domains (https:/ / www.ncbi.nlm.nih.gov/Structure/ cdd/wrpsb.cgi, accessed on 20 August 2021), and a schematic diagram was generated by Adobe Illustrator CC 2019 software (Adobe, San Jose, CA, USA). 


\subsection{2. qPCR Analysis}

Ten tissues (i.e., kidney, gill, liver, spleen, brain, heart, posterior intestine, skin, fin, and barbel) were sampled from WT loach, large-scale loach, zebrafish, and yellow catfish. All samples were used for qPCR analysis, and the expression levels of $f n 1 b$ in different tissues from the four kinds of fishes were analyzed by MetaboAnalyst5.0 (https:/ / www. metaboanalyst.ca, accessed on 20 August 2021). In addition, gills (aquatic breathing organ) were sampled from rice-field eel and snakehead fish. The air-breathing organs, oropharyngeal cavity and suprabranchial organ, were dissected from rice-field eel and snakehead fish, respectively. All samples were used for qPCR analysis, and $\beta$-actin was used as the reference gene. All of the primer sequences for qPCR are listed in Table S3.

\subsubsection{In Situ Hybridization}

To locate the $f n 1 b$ gene in the loach barbel, a specific Dig-labeling antisense RNA probe was synthesized by using T7 in vitro transcriptional polymerase with a DIG RNA labeling kit (Roche Molecular Biochemicals, Mannheim, Germany). The probe for $f n 1 b$ location was amplified from the cDNA pool using appropriate primers (Table S3). A healthy WT loach was selected for reproduction. Development of embryos occurred at $26^{\circ} \mathrm{C}$, and staging was determined by hours post fertilization. Living loach embryos were sampled at different developmental stages: $12 \mathrm{~h}$ post fertilization (hpf), $24 \mathrm{hpf}, 36 \mathrm{hpf}, 5$ days post fertilization (dpf), and $12 \mathrm{dpf}$. All embryos were fixed in $4 \%(w / v)$ paraformaldehyde. Then, whole-mount in situ hybridization (WISH) of loach embryos was conducted according to a previously described method [30]. As for tissue in situ hybridization (TISH), barbels from WT loach were sampled and fixed in $4 \%(w / v)$ paraformaldehyde. The procedure of TISH was performed as described previously [52].

\subsection{Effects of fn1b-Depletion on Loach Barbel Air-Breathing}

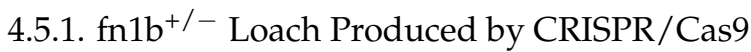

The $f n 1 b$-depletion loach was generated using CRISPR/Cas9 technology. The Cas 9 targeting site design website was used to design the appropriate targeting site (http: / / zifit. partners.org/ZiFi/, accessed on 20 August 2021). Following all design principles, the Cas9 targeting site "GATTGTACCTGCATTGGGTCAGG" was determined. In vitro transcription of Cas9 RNA and gRNA was based on the standards of relevant research [53], and detailed construction methods were performed as previously described [54]. After microinjection, the genomic DNA was isolated from six randomly selected fertilized embryos. Next, the target genome region was amplified and sequenced. The primers for mutation analysis are listed in Table S3. Once the mutation was confirmed in injected embryos, the remaining ones were raised to adulthood, and the mutant ones (F0 generation) were outcrossed with WT loach to produce the F1 generation. The heterozygous F1 generation with the same mutation sequences was self-crossed. During the period, the survival rates of loach fertilized eggs were recorded. Since homozygous mutants (lethal) could not be obtained, the $f n 1 b^{+/-}$loach ( $f n 1 b$-depletion loach, MT) was used for further analysis. The expression levels of $f n 1 b$ in barbels of WT and MT loach were measured.

\subsubsection{Histological Observations of Barbels from WT and MT Loach}

The barbels were collected from WT and MT loach. H\&E staining of a barbel tissue section was performed, and barbel blood-gas diffusion distances were measured.

\subsubsection{Chronic Hypoxia Experiment}

A chronic hypoxic experiment was performed to explore the effects of $f n 1 b$-depletion on loach air-breathing (Figure S5D). Three WT and three MT loaches were placed into a $2 \mathrm{~L}$ conical flask containing $1.5 \mathrm{~L}$ of water. We sealed the conical flask to continuously reduce the dissolved oxygen level, and the water dissolved oxygen level was monitored by a dissolved oxygen detector in real time until two loaches fainted. During this process, water dissolved oxygen levels, air-breathing frequencies, and asphyxia points were recorded. 
At the same time, the swimming behaviors of the loaches were observed. Three parallel experiments were carried out.

\subsection{Statistical Analysis}

All data were presented as the mean \pm standard deviation (SD). Statistical analyses were performed using SPSS 26.0 software (IBM Analytics, Richmond, VA, USA). For twogroup comparison, a $t$-test was performed. One-way ANOVA was performed followed by Tukey's test for multiple comparisons. A $p$-value $<0.05$ was considered significant, whereas $p<0.01$ indicated a very significant difference.

Supplementary Materials: The following are available online at https: / www.mdpi.com/article/ 10.3390/ijms222111928/s1: Figure S1. Observations of H\&E-staining longitudinal sections of loach barbels; Figure S2. Functional annotations of DEGs in barbels between T-XU and C-XU groups (T-XU vs. C-XU); Figure S3. Schematic diagram of the conserved domains of the deduced amino-acid sequences of fibronectin 1 gene $(f n 1)$ in loach $M$. anguillicaudatus, compared with three other fish species (D. rerio, C. argus, and M. albus); Figure S4. fn1b-depletion loach produced by CRISPR/Cas9 technology; Figure S5. Four treatments used in this study. Table S1. Blood-gas diffusion distances of air-breathing organs in different air-breathing fishes; Table S2. Quality and mapped ratio of clean reads; Table S3. Primers used in this study.

Author Contributions: Formal analysis, S.H.; funding acquisition, J.G. and X.C.; investigation, B.S., S.H., L.H. and L.Y.; project administration, J.G.; resources, B.S.; software, S.H.; supervision, J.G. and X.C.; validation, L.H. and L.Y.; writing—original draft, B.S.; writing—review and editing, X.C. All authors have read and agreed to the published version of the manuscript.

Funding: This study was supported by Fundamental Research Funds for the Central Universities (2662020SCPY002) and the Ministry of Agriculture and Rural Affairs of the People's Republic of China (Project Title: Conservation and proliferation of loach germplasm resources in the Yangtze River Basin).

Institutional Review Board Statement: This study was approved by the Committee on the Ethics of Animal Experiments of Huazhong Agricultural University (HZAUFI-2021-0023). Fish sampling was performed under anesthesia with MS-222 (tricaine methanesulfonate, Sigma, USA) at the concentration of $200 \mathrm{mg} / \mathrm{L}$ to minimize suffering.

Informed Consent Statement: Not applicable.

Data Availability Statement: Data from this study can be provided upon request.

Acknowledgments: We thank Wang from the University of California Davis for revising the English writing of this manuscript. We thank the two anonymous reviewers for their helpful comments and suggestions.

Conflicts of Interest: The authors declare no conflict of interest.

Data Accessibility: Raw sequencing data were deposited in the NCBI Sequence Read Archive (SRA) under accession number PRJNA743945.

\section{References}

1. Ma, X.; Su, B.; Bangs, M.; Alston, V.; Backenstose, N.; Simora, R.M.; Wang, W.; Xing, D.; Li, S.; Ye, Z. Comparative Genomic and Transcriptomic Analyses Revealed Twenty-Six Candidate Genes Involved in the Air-Breathing Development and Function of the Bighead Catfish Clarias macrocephalus. Mar. Biotechnol. 2021, 23, 90-105. [CrossRef]

2. Wang, K.; Wang, J.; Zhu, C.; Yang, L.; Wang, W. African lungfish genome sheds light on the vertebrate water-to-land transition. Cell 2021, 5, 1362-1376. [CrossRef]

3. Dobson, G.E. Notes on the Respiration of some Species of Indian Freshwater Fishes. Proc. Zool. Soc. Lond. 1874, 42, 312-321. [CrossRef]

4. Nelson, J.A. Breaking wind to survive: Fishes that breathe air with their gut. J. Fish. Biol. 2014, 84, 554-576. [CrossRef]

5. Munshi, J.; Olson, K.R.; Ojha, J.; Ghosh, T.K. Morphology and vascular anatomy of the accessory respiratory organs of the air-breathing climbing perch, Anabas testudineus (Bloch). Dev. Dyn. 2010, 176, 321-331. [CrossRef]

6. Johansen, K. Air breathing in Fishes. Fish. Physiol. 1970, 4, 361-411. [CrossRef] 
7. Huebner, E.; Chee, G. Histological and ultrastructural specialization of the digestive tract of the intestinal air breather hoplosternum thoracatum (teleost). J. Morphol. 1978, 157, 301-327. [CrossRef]

8. Satora, L. Histological and ultrastructural study of the stomach of the air-breathing Ancistrus multispinnis (Siluriformes, Teleostei). Can. J. Zool. 1998, 76, 83-86. [CrossRef]

9. Liu, Y.Q.; Li, X.H.; Zhao, J.R.; Wang, Z.J. Effect of Intestinal Air-breathing Restriction on Respiratory Metabolism and Antioxidant Capability of Loach (Paramisgurnus dabryanus). Chin. J. Zool. 2017, 5, 857-864. [CrossRef]

10. Hughes, G.M.; Singh, B.N. Respiration in an air-breathing fish, the climbing perch Anabas testudineus. J. Exp. Biol. 1970, 53, 265-280. [CrossRef]

11. Hakim, A.; Datta Munshi, J.S.; Hughes, G.M. Morphometries of the respiratory organs of the Indian green snake-headed fish. Channa punctata. J. Zool. 1978, 184, 519-543. [CrossRef]

12. Yadav, A.N.; Singh, B.R. The gut of an intestinal air-breathing fish, Lepidocephalus guntea (Ham). Arch. Biol. 1980, 91, 413-422.

13. Weibel, E.R. Morphological basis of alveolar-capillary gas exchange. Physiol. Rev. 1973, 53, 419-495. [CrossRef] [PubMed]

14. García, L. Histology, ultrastructure and immunohistochemistry of the respiratory organs in non mammalian vertebrates. Biochem. Educ. 1995, 10, 125. [CrossRef]

15. Frommel, A.Y.; Kwan, G.T.; Prime, K.J.; Tresguerres, M.; Brauner, C.J. Changes in gill and air-breathing organ characteristics during the transition from waterto air-breathing in juvenile Arapaima gigas. J. Exp. Zool. A Ecol. Integr. Physiol. 2021, 42, 312-321. [CrossRef]

16. Bi, X.; Wang, K.; Yang, L.; Pan, H.; Jiang, H.; Wei, Q.; Fang, M.; Yu, H.; Zhu, C.; Cai, Y. Tracing the genetic footprints of vertebrate landing in non-teleost ray-finned fishes. Cell 2021, 5, 1377-1391. [CrossRef] [PubMed]

17. Banerjee, B.; Koner, D.; Hasan, R.; Saha, N. Molecular characterization and ornithine-urea cycle genes expression in air-breathing magur catfish (Clarias magur) during exposure to high external ammonia. Genomics 2019, 112, 2247-2260. [CrossRef]

18. Li, N.; Bao, L.; Zhou, T.; Yuan, Z.; Liu, S.; Dunham, R.; Li, Y.; Wang, K.; Xu, X.; Jin, Y. Genome sequence of walking catfish (Clarias batrachus) provides insights into terrestrial adaptation. BMC Genom. 2018, 19, 952. [CrossRef] [PubMed]

19. Luo, W.W.; Cao, X.J.; Xu, X.; Huang, S.Q.; Liu, C.S.; Tomljanovic, T. Developmental transcriptome analysis and identification of genes involved in formation of intestinal air-breathing function of Loach, Misgurnus anguillicaudatus. Sci Rep. 2016, 6, 31845. [CrossRef]

20. Huang, S.Q.; Cao, X.J.; Tian, X. Transcriptomic Analysis of Compromise Between Air-Breathing and Nutrient Uptake of Posterior Intestine in Loach (Misgurnus anguillicaudatus), an Air-Breathing Fish. Mar. Biotechnol. 2016, 18, 1-13. [CrossRef]

21. Jiang, Y.; Feng, S.; Xu, J.; Zhang, S.; Li, S.; Sun, X.; Xu, P. Comparative transcriptome analysis between aquatic and aerial breathing organs of Channa argus to reveal the genetic basis underlying bimodal respiration. Mar. Genom. 2016, 29, 89-96. [CrossRef]

22. Yang, Y.J. Comparative Transcriptome Analysis of Catfish Swimbladder Reveals Expression Signatures in Chamber Formation and their Response to Low Oxygen Stress. Ph.D. Dissertation, Auburn University, Auburn, AL, USA, 2018. Available online: http:/ / hdl.handle.net/10415/6301 (accessed on 25 August 2021).

23. Norris, K.E. Growth, Fecundity, and Diet of Oriental Weatherloach Misgurnus Anguillicaudatus in the Chicago Area Waterways; Western Illinois University: Macomb, IL, USA, 2015. [CrossRef]

24. Mcmahon, R. Resriatory physiology of intestinal air breathing in the teleost fish Misgurnus anguillicaudatus. J. Exp. Biol. 1987, 133, 371-393. [CrossRef]

25. Huang, S.Q.; Cao, X.J.; Tian, X.; Wang, W.M. High-Throughput Sequencing Identifies MicroRNAs from Posterior Intestine of Loach (Misgurnus anguillicaudatus) and Their Response to Intestinal Air-Breathing Inhibition. PLoS ONE 2016, 11, e0149123. [CrossRef]

26. Luo, W.W.; Liang, X.; Huang, S.Q.; Cao, X.J. Molecular cloning, expression analysis and miRNA prediction of vascular endothelial growth factor A (VEGFAa and VEGFAb) in pond loach Misgurnus anguillicaudatus, an air-breathing fish. Comp. Biochem. Physiol. B Biochem. Mol. Biol. 2016, 202, 39-47. [CrossRef]

27. Leclair, E.E.; Jacek, T. Methods for the Study of the Zebrafish Maxillary Barbel. J. Vis. Exp. 2009, 33, 1494-1499. [CrossRef] [PubMed]

28. Leclair, E.E.; Jacek, T.; Bruce, R. Development and regeneration of the zebrafish maxillary barbel: A novel study system for vertebrate tissue growth and repair. PLoS ONE 2010, 5, e8737. [CrossRef] [PubMed]

29. Duszynski, R.J.; Topczewski, J.; Leclair, E.E. Divergent requirements for FGF signaling in zebrafish maxillary barbel and caudal fin regeneration. Dev. Growth Differ. 2013, 55, 282-300. [CrossRef]

30. Zhou, T.; Li, N.; Jin, Y.; Zeng, Q.; Prabowo, W.; Liu, Y.; Tian, C.; Bao, L.; Liu, S.; Yuan, Z. Chemokine C-C motif ligand 33 is a key regulator of teleost fish barbel development. Proc. Nat. Acad. Sci. USA 2018, 115, 5018-5027. [CrossRef] [PubMed]

31. Li, J.X.; Yang, C.; Huang, L.F.; Zeng, K.; Cao, X.J. Inefficient ATP synthesis by inhibiting mitochondrial respiration causes lipids to decrease in MSTN-lacking muscles of loach Misgurnus anguillicaudatus. Funct. Integr. Genom. 2019, 19, 889-900. [CrossRef] [PubMed]

32. Jayaram, K.C. Functional responses of catfish barbels. Bull. Zool. Surv. India 1978, 1, 77-80.

33. Haynes, G.D.; Gongora, J.; Gilligan, D.M.; Grewe, P.; Moran, C.; Nicholas, F.W. Cryptic hybridization and introgression between invasive Cyprinid species Cyprinus carpio and Carassius auratus in Australia: Implications for invasive species management. Anim. Conserv. 2012, 15, 83-94. [CrossRef] 
34. Olson, K.R.; Roy, P.K.; Ghosh, T.K.; Munshi, J. Microcirculation of gills and accessory respiratory organs from the air-breathing snakehead fish, Channa punctata, C. gaucha and C. marulius. Anat. Rec. 2010, 238, 92-107. [CrossRef]

35. Niva, B.; Ojha, J.; Munshi, J. Morphometrics of the respiratory organs of an estuarine goby, Boleophthalmus boddaerti. Japan J. Ichthyol. 1981, 27, 316-326. [CrossRef]

36. Wu, C.L.; Dicks, A.; Steward, N.; Tang, R.; Guilak, F. Single cell transcriptomic analysis of human pluripotent stem cell chondrogenesis. Nat. Commun. 2021, 12, 362. [CrossRef] [PubMed]

37. Liu, L.; Zhang, R.; Wang, X.; Zhu, H.; Tian, Z. Transcriptome analysis reveals molecular mechanisms responsive to acute cold stress in the tropical stenothermal fish tiger barb (Puntius tetrazona). BMC Genom. 2020, 21, 737. [CrossRef]

38. Le, A.T.; Stainier, D. Fibronectin regulates epithelial organization during myocardial migration in zebrafish. Dev. Cell 2004, 6 , 371-382. [CrossRef]

39. Wijelath, E.S. Novel Vascular Endothelial Growth Factor Binding Domains of Fibronectin Enhance Vascular Endothelial Growth Factor Biological Activity. Circ. Res. 2002, 91, 25-31. [CrossRef]

40. George, E.L.; Baldwin, H.S.; Hynes, R.O. Fibronectins are essential for heart and blood vessel morphogenesis but are dispensable for initial specification of precursor cells. Blood 1997, 90, 3073-3081. [CrossRef]

41. Mcdougal, B.A. A Comparative Study of Tissue Oxygen Consumption Correlated with the Molting Cycle in the Fresh Water Crayfish, Procambarus Simulans; Texas Tech University: Lubbock, TX, USA, 1965; Available online: http://hdl.handle.net/2346/1 (accessed on 25 August 2021).

42. Gordon, M.S.; Chin, H.G.; Vojkovich, M. Energetics of swimming in fishes using different methods of locomotion: I. Labriform swimmers. Fish Physiol. Biochem. 1989, 6, 341-352. [CrossRef]

43. Zhao, Q.; Liu, X.; Collodi, P. Identification and characterization of a novel fibronectin in zebrafish. Exp. Cell Res. 2001, 268, 211-219. [CrossRef]

44. Chang, Y.; Shiu, J.; Chuang, W. Design, Structure Determination, and Biological Evaluation of Potent Integrin Alpha5beta1Specific Antagonist Using the Ninth and Tenth Module of Fibronectin Type III Domain. Ph.D. Dissertation, National Cheng Kung University, Taiwan, 2013.

45. Cao, X.J.; Wang, W.M. Histology and Mucin Histochemistry of The Digestive Tract of Yellow Catfish, Pelteobagrus fulvidraco. Anat. Histol. Embryol. 2010, 38, 254-261. [CrossRef] [PubMed]

46. Kim, D.; Langmead, B.; Salzberg, S.L. HISAT: A fast spliced aligner with low memory requirements. Nat. Methods 2015, 12, 357-360. [CrossRef] [PubMed]

47. Pertea, M.; Pertea, G.M.; Antonescu, C.M.; Chang, T.C.; Mendell, J.T.; Salzberg, S.L. StringTie enables improved reconstruction of a transcriptome from RNA-seq reads. Nat. Biotechnol. 2015, 33, 290-295. [CrossRef]

48. Dewey, C.N.; Li, B. RSEM: Accurate transcript quantification from RNA-Seq data with or without a reference genome. BMC Bioinform. 2011, 12, 323. [CrossRef]

49. Love, M.I.; Huber, W.; Anders, S. Moderated estimation of fold change and dispersion for RNA-seq data with DESeq2. Genome Biol. 2014, 15, 550. [CrossRef]

50. Chen, X.; Mao, X.; Huang, J.; Yang, D.; Wu, J.; Dong, S.; Lei, K.; Ge, G.; Li, C.Y.; Wei, L. KOBAS 2.0: A web server for annotation and identification of enriched pathways and diseases. Nucleic Acids Res. 2011, 39, 316-322. [CrossRef]

51. Liu, J.X.; Zhai, Y.H.; Gui, J.F. Molecular characterization and expression pattern of AFPIV during embryogenesis in gibel carp (Carassiu auratus gibelio). Mol. Biol. Rep. 2009, 36, 2011-2018. [CrossRef]

52. Randilini, A.; Fujikawa, K.; Shibata, S. An in situ hybridization study of decorin and biglycan mRNA in mouse osteoblasts in vivo. Anat. Sci. Int. 2021, 96, 265-272. [CrossRef] [PubMed]

53. Moreno-Mateos, M.A.; Vejnar, C.E.; Beaudoin, J.D.; Fernandez, J.P.; Mis, E.K.; Khokha, M.K.; Giraldez, A.J. CRISPRscan: Designing highly efficient sgRNAs for CRISPR-Cas9 targeting in vivo. Nat. Methods 2015, 12, 982-988. [CrossRef]

54. Sun, S.X.; Ren, T.Y.; Li, X.; Cao, X.J.; Gao, J. Polyunsaturated fatty acids synthesized by freshwater fish: A new insight to the roles of elovl2 and elovl5 in vivo. Biochem. Biophys. Res. Commun. 2020, 532, 414-419. [CrossRef] 